\title{
Sub-10 fs pulse characterization using spatially encoded arrangement for spectral phase interferometry for direct electric field reconstruction
}

\author{
Adam S. Wyatt and Ian A. Walmsley \\ Clarendon Laboratory, University of Oxford, Parks Road, Oxford, OX1 3PU, UK
}

Gero Stibenz and Günter Steinmeyer

Max-Born-Institut für Nichtlineare Optik und Kurzzeitspektroskopie, Max-Born-Strasse 2A, D-12489 Berlin, Germany

\begin{abstract}
Received January 18, 2006; accepted March 14, 2006; posted March 5, 2006 (Doc. ID 67345)
We demonstrate an extremely accurate method for measuring ultrabroadband, sub- $10 \mathrm{fs}$ pulses even if they exhibit a highly modulated spectrum, space-time coupling, or both. The method uses a spatially encoded arrangement for spectral phase interferometry for direct electric field reconstruction, which allows a zero additional phase measurement to be performed with a relatively low signal-to-noise ratio in real time and single shot. (C) 2006 Optical Society of America
\end{abstract}

OCIS codes: $320.320,320.7100,120.3180,120.5050$.

Intense sub-10 fs pulses are used in a broad range of applications. For example, in high-harmonic generation ${ }^{1}$ and in the production of isolated attosecond pulses, ${ }^{2}$ it is necessary to have accurate, full characterization of the driving pulse to understand the physics of the generation process in detail or to be able to compensate the spectral phase to generate Fourier-transform-limited pulses. The most widespread characterization techniques for ultrashort optical pulses are frequency-resolved optical gating $(\text { FROG })^{3}$ and spectral phase interferometry for direct electric field reconstruction (SPIDER). ${ }^{4}$ Although these techniques are proven characterization methods in the sub-10 fs range, ${ }^{5,6}$ their application can become challenging or even impossible under certain conditions. These include cases when (1) the spectrum is ultrabroadband (e.g., over an octave), (2) the spectrum is highly modulated, (3) the pulse exhibits space-time coupling ${ }^{7}$ (STC), and (4) it is desirable to have single-shot acquisition. In this Letter we discuss a spatially encoded arrangement (SEA) for SPIDER $^{8}$ as a method to overcome these problems.

The difficulties in measuring sub-10 fs pulses lie in the method of encoding the pulse-shape information in conventional FROG and SPIDER. FROG requires a $2 \mathrm{D}$ data set that encodes a $1 \mathrm{D}$ field. It can operate near the spectral sampling limit (given by the Whitaker-Shannon theorem) and uses an iterative deconvolution algorithm to reconstruct the field. A FROG apparatus is usually configured to spectrally and temporally oversample the pulse so that it can measure complex pulse-shapes with little adjustment. However, the size of data and convergence time increase nonlinearly with the pulse complexity. Single-shot acquisition can be accomplished only when no STC is present. SPIDER records a 1D data set with a direct, linear reconstruction algorithm. Since the phase is encoded in the spacing of spectral fringes, SPIDER oversamples the spectrum significantly. If the spectral amplitude has a lot of struc- ture, very high spectral resolution may be necessary to resolve the fringes, especially in regions of low spectral intensity, if phase errors are to be avoided. However, SPIDER can measure STC and perform single-shot acquisition simultaneously, with rapid reconstruction.

In this Letter we present an extremely accurate measurement of ultrabroadband pulses $(>350 \mathrm{~nm}$ spectral coverage at $800 \mathrm{~nm}$ ) using SEA-SPIDER. To our knowledge, this is currently the only technique that can measure near-octave spanning pulses with such accuracy and simultaneously overcome all four problems stated in the first paragraph. We illustrate how SEA-SPIDER eliminates the problems presented with measuring sub-10 fs pulses with conventional SPIDER while still retaining its many advantages over other techniques.

In SEA-SPIDER the test pulse upconverts with two spatially tilted, temporally delayed, and highly chirped pulses (Fig. 1). During the interaction time, the test pulse sees two different quasimonochromatic frequencies, generating two spectrally sheared replicas that are imaged onto the entrance slit of a $2 \mathrm{D}$ imaging spectrometer. The tilt between these two signal pulses induces a fringe pattern in the spatial dimension of the spectrometer. The interferogram is represented by

$$
\begin{aligned}
I(\omega, x)= & |E(\omega, x)|^{2}+|E(\omega-\Omega, x)|^{2} \\
& +2|E(\omega, x)||E(\omega-\Omega, x)| \\
& \times \cos [\phi(\omega, x)-\phi(\omega-\Omega, x)+K x],
\end{aligned}
$$

where $x$ is the spatial position across the beam, $K$ is the difference in the mean wave vector between the two signal arms, and $\omega$ is the spectral frequency in units of radians per second.

The SEA-SPIDER apparatus used to measure sub$10 \mathrm{fs}$ pulses is shown in Fig. 2. The short pulse duration and large bandwidth allow the use of a dispersive glass block instead of a grating stretcher to gen- 


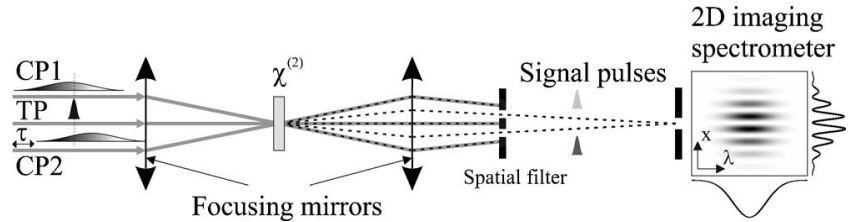

Fig. 1. SEA-SPIDER concept: TP, test pulse; CP1/CP2, chirped pulse $1 / 2 ; \tau$, time delay.

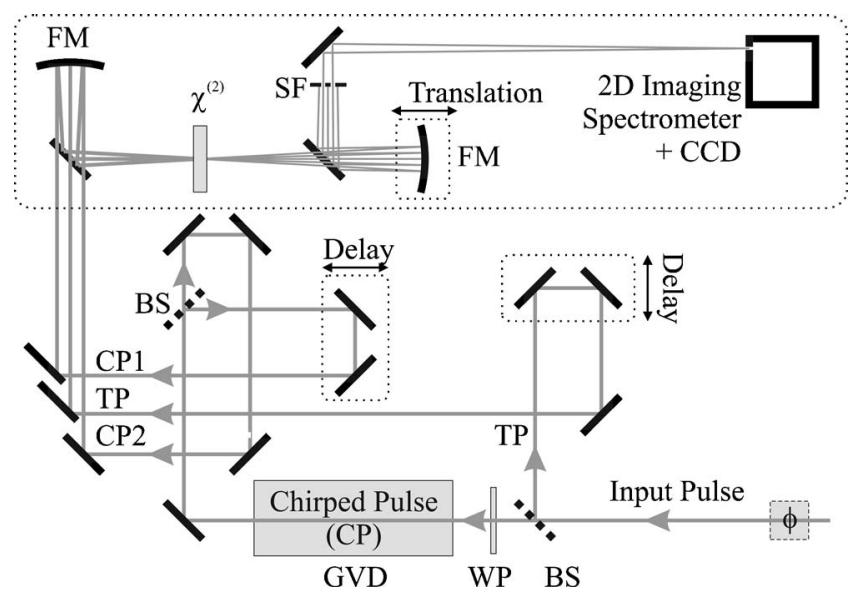

Fig. 2. SEA-SPIDER setup: BS, beam splitter; WP, $\lambda / 2$ wave plate; GVD, dispersive glass block (10 cm SF10); FM, focusing mirror $(f=100 \mathrm{~mm}) ; \chi^{(2)}$, nonlinear crystal $(30 \mu \mathrm{m}$ $\beta$-barium borate, type II); SF, spatial filter; $\phi$, optional additional phase.

erate the chirped pulses. The delay between the two chirped pulses sets the shear, whereas the delay between the test pulse and chirped pulses sets the average central frequency for the upconversion. Note that none of these delays requires interferometric stability. Furthermore, as SEA-SPIDER uses two stretched pulses and only one short test pulse, there is no danger of inducing a phase imbalance upon replication of the short pulse, ensuring that the test pulse acquires zero additional spectral phase. ${ }^{9}$ This is essential for measuring pulses of such large bandwidth because only a small amount of dispersion is necessary to significantly stretch the pulse. The focusing mirror after the crystal is translated to ensure the interferogram is imaged correctly (i.e., the two signal pulses spatially overlap perfectly).

To test the apparatus, we measured a pulse with a spectral range from 650 to $1000 \mathrm{~nm}$ (i.e., $6.5 \mathrm{fs}$ FWHM Fourier-transform-limited duration) directly from an oscillator (Femtolaser Rainbow). The pulse was sampled on a 2D imaging spectrometer (Acton SP-150) with a grating groove spacing of 150 lines/mm and detected on a $1000 \times 1000 \mathrm{CCD}$ array (Pulnix 1040). The spectrometer's range was measured to be $3.38 \mathrm{rad} / \mathrm{fs}$, which is enough to support single-cycle pulses at this wavelength. The integration time and update rate was $30 \mathrm{~Hz}$. As the data were fully contained within $150 \times 300$ pixels, kilohertz acquisition should be possible. The inversion routine is slower than for SPIDER but can still run at several hertz. The input pulse energy was $<3.8 \mathrm{~nJ}$ $(<300 \mathrm{~mW}$ at $1 \mathrm{kHz})$, although lower energies could also be measured.
SEA-SPIDER measures both the spectrum and phase at every point in a slice across the beam and thus can measure STC directly. ${ }^{8}$ However, in the absence of any STC, the 2D interferogram is very intuitive: the contours of the fringes directly map the spectral phase gradient (i.e., the chirp). Figure 3 shows four interferograms illustrating this intuitive nature. If the shear is set to zero (by setting the delay between the two chirped pulses to zero), then the only term left inside the cosine of Eq. (1) is $K x$. As this is only a linear function of $x$ and is independent of wavelength (within the CCD resolution), the fringes appear horizontal [Fig. 3(a)]. To measure the test pulse phase, a shear of $27 \mathrm{mrad} / \mathrm{fs}$ was introduced between the two signal pulses (by delaying one chirped pulse with respect to the other). This is enough to sample a pulse with compact temporal support of $230 \mathrm{fs}$. The horizontal fringes [Fig. 3(b)] result from no group-velocity dispersion (GVD), but the small modulation in the fringe contours (dashed curve) results from higher-order dispersion. This is confirmed by the full reconstruction [Fig. 4(b)]. Figures 3(c) and 3(d) show the SEA-SPIDER interferogram for positive (GVD $\sim+350 \mathrm{fs}^{2} / \mathrm{rad}$ ) and negative chirp (GVD $\sim-120 \mathrm{fs}^{2} / \mathrm{rad}$ ), respectively. This is shown in the traces by the positive or negative linear slope of the fringe contours. Figure 3(c) also exhibits a slight negative curvature, which corresponds to small negative third-order phase. Finally, the interference traces can show any discontinuities in the phase. The negative chirp in Fig. 3(d) was generated by reflecting the pulse off a chirped mirror pair. However, the chirped-mirror bandwidth was less than the pulse spectrum; hence there are discontinuities in the fringe pattern at approximately 390 and $450 \mathrm{~nm}$. This is useful when identifying optics with limited bandwidth.

To test the accuracy of the apparatus, we added known dispersion to the test pulse by passing it through $1 \mathrm{~cm}$ of fused silica before the SEA-SPIDER apparatus. We compared the measured phase of the silica with that calculated by using the Sellmeier equations. The two phase profiles are shown in Fig. 4(a) with the oscillator spectrum. The agreement is excellent; the two curves are almost indistinguishable. To quantify the difference we use the pulse er-

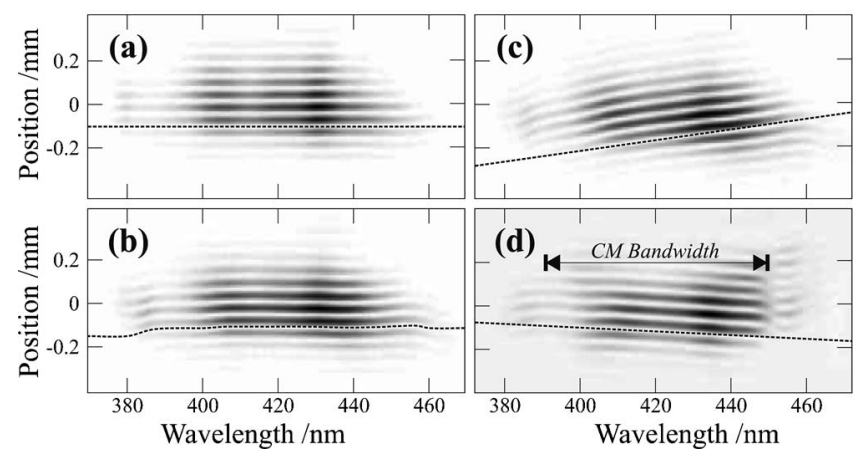

Fig. 3. SEA-SPIDER interferograms, dashed lines show fringe pattern: (a) calibration trace (i.e., zero shear); (b) SEA-SPIDER measurement of the Rainbow oscillator; (c) SEA-SPIDER measurement of a positive chirped pulse; (d) SEA-SPIDER measurement of a negative chirped pulse. 


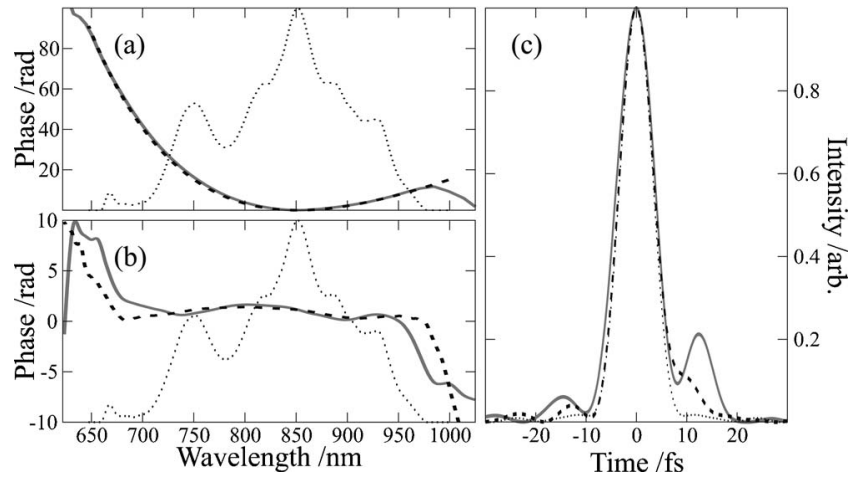

Fig. 4. Pulse reconstruction: (a) measured (solid gray curve) and calculated (dashed black curve) phase of $1 \mathrm{~cm}$ fused silica; (b) oscillator phase reconstruction from SEASPIDER (solid gray curve) and SPIDER (dashed black curve); (c) temporal reconstruction from SEA-SPIDER (solid gray curve), SPIDER (dashed black curve) and Fourier-transform-limited pulse (dotted black curve).

ror for normalized fields, ${ }^{10}$ calculated to be $\epsilon_{\text {silica }}$ $=0.016$. Note that the excellent agreement holds over the whole range where the spectrum is greater than the background (a spectral range of $\simeq 350 \mathrm{~nm}$ ), even in areas of low spectral intensity (e.g., $\lambda<725 \mathrm{~nm}$, where the spectrum is less than $1 \%$ of the maximum giving a dynamic range greater than $20 \mathrm{~dB}$ ). The reconstructed phase was verified by comparing it with the phase measured by using the conventional SPIDER.

Figure 3(b) shows the SEA-SPIDER phase, SPIDER phase, and the pulse spectrum. The pulse error between the two different measurements was calculated to be $\epsilon_{\text {Rainbow }}=0.11$, which again shows good agreement. The two measured phase profiles have excellent agreement over the range from 725 to $950 \mathrm{~nm}$. Outside this range, errors occur in the SPIDER reconstruction for the following reasons: (1) the spectrum varies almost as fast as the fringes, and (2) the spectral intensity is low. As a result, it is not possible to resolve the fringes in the SPIDER interferogram. In contrast, the SEA-SPIDER fringes are in the spatial dimension, thus the shape of the spectrum does not affect the ability to resolve these fringes. The signal-to-noise ratio requirement for SEA-SPIDER is lower than for conventional SPIDER because the inversion algorithm is a $2 \mathrm{D}$ Fourier transform, which permits a higher noise rejection when filtering. The data plotted in Fig. 4 are the pulse shape at a single point in the beam (i.e., with no spatial averaging). The large phase variation from the SEA-SPIDER reconstruction at the edge of the spectrum is real and can probably be attributed to a limited bandwidth of the chirped mirrors used inside the oscillator. We also verified there was no significant STC for this oscillator.

The temporal pulse profile was calculated and is plotted in Fig. 4(c) by using the measured spectrum and zero phase, SEA-SPIDER phase, or SPIDER phase. The FWHM pulse durations were calculated to be $6.5,8.6$, and $7.9 \mathrm{fs}$, respectively. Because of the large bandwidth of the pulse, very small deviations of air path lengths, as well as differences of the intrinsic dispersion of the two setups, can significantly affect the results. This explains the small differences in measured pulse duration, but not the more significant subpulse in the SEA-SPIDER reconstruction, which can be attributed to the phase at the edge of the spectrum that SPIDER could not recover. Thus it is clear that SEA-SPIDER can resolve features that can be important in certain applications when SPIDER cannot.

In conclusion, we have demonstrated the capability of SEA-SPIDER for measuring ultrabroadband pulses (i.e., a spectral bandwidth from $350 \mathrm{~nm}$, or sub-10 fs Fourier-transform-limited duration, FWHM). The setup provides a zero additional phase measurement, which is extremely accurate over the whole bandwidth, even in regions of low spectral intensity. The phase accuracy is independent of spectral shape, with the only requirement being that the spatial fringe contrast be greater than the noise, and the decoupling of the shear from the encoding of the fringes allow a wider variety of pulse shapes to be measured. STC can be measured directly without the need to assume a functional form for the coupling. In the absence of STC, the interference traces show intuitive behavior, thus manual phase adjustment can be performed without reconstruction. The pulses can be sampled at the Whittaker-Shannon limit, allowing the use of a low-dispersion spectrometer or the measurement of highly chirped pulses. The apparatus was shown to be very sensitive and hence can be used inline with existing experiments with amplified pulses by taking only $<1 \%$ of the pulse power, or it can be used with single-shot measurements on a $1 \mathrm{kHz}$ system provided a suitable camera is used.

A. S. Wyatt and I. A. Walmsley acknowledge useful discussions with A. Monmayrant. This work was funded in part by the Research Councils UK as part of the Basic Technology Program, under the aegis of the UK Attoscience Consortium. A. S. Wyatt's e-mail address is a.wyatt1@physics.ox.ac.uk.

\section{References}

1. M. Lewenstein, P. Balcou, M. Y. Ivanov, A. L'Huillier, and P. B. Corkum, Phys. Rev. A 49, 2117 (1994)

2. M. Hentschel, R. Kienberger, C. Spielmann, G. A. Reider, N. Milosevic, T. Brabec, P. Corkum, U. Heinzmann, M. Drescher, and F. Krausz, Nature 414, 509 (2001).

3. R. Trebino and D. J. Kane, J. Opt. Soc. Am. A 10, 1101 (1993).

4. C. Iaconis and I. A. Walmsley, Opt. Lett. 23, 792 (1998).

5. A. Baltuška, M. S. Pshenichnikov, and D. A. Wiersma, Opt. Lett. 23, 1474 (1998).

6. L. Gallmann, D. H. Sutter, N. Matuschek, G. Steinmeyer, U. Keller, C. Iaconis, and I. A. Walmsley, Opt. Lett. 24, 1314 (1999).

7. C. Dorrer and I. A. Walmsley, Opt. Lett. 27, 1947 (2002)

8. E. M. Kosik, A. S. Radunsky, I. A. Walmsley, and C. Dorrer, Opt. Lett. 30, 326 (2005).

9. P. Baum, S. Lochbrunner, and E. Riedle, Opt. Lett. 29, 210 (2004).

10. C. Dorrer and I. A. Walmsley, J. Opt. Soc. Am. B 19, 1019 (2002). 